\title{
FIELD OF A RADIATION DISTRIBUTION
}

\author{
I. H. Dwivedi币 \\ Theoretical Astrophysics Group \\ Tata Institute of Fundamental Research \\ Homi Bhabha Road, Colaba, Bombay 400005, India.
}

\begin{abstract}
General relativistic spherically symmetric matter field with a vanishing stress energy scalar is analyzed. Procedure for generating exact solutions of the field equations for such matter distributions is given. It is further pointed out that all such type I spherically symmetric fields with distinct eignvalues in the radial two space can be treated as a mixture of isotropic and directed radiations. Various classes of exact solutions are given. Junction conditions for such a matter field to the possible exterior solutions are also discussed.
\end{abstract}

\section{INTRODUCTION}

Physical aspects of nature can be formulated in the form of a mathematical model comprising of a set of differential equations. Solutions to this set of differential equations are used not only to confirm the validity of the proposed theory by way of comparing its prediction for a particular known phenomena in nature, but also to predict possibly new unknown or even yet to be discovered natural phenomena. General relativity, despite being the most elegant mathematical theory of gravitation, has one of the most complicated set of differential equations and getting exact solutions describing a realistic physical scenario is a difficult task. Though after the discovery of the first exact solution, namely the Schwarzschild solution, numerous sets and classes of exact solutions of the field equations are given in the literature ${ }^{1}$, still only very few of them are physically realistic. Perhaps this may be the reason that despite being accepted as the best theory of gravitation and the fact that gravity is the most dominant force at present stage in our universe the tests of relativity theory as a true

\footnotetext{
${ }^{1}$ E-mail : dwivedi@tifrvax.tifr.res.in
} 
theory of gravitation are basically three coming from the first discovered and most widely used vacuum Schwarzschild solution. The study of various solutions of the fields equations from the mathematical point of view has its own merit and from that prospective there is a sea of solutions to the field equation. However, we still do not have exact solutions for most physical situations. The very few exact solutions that do describe some physically realistic situations are Schwarzschild space-time and Ker space-time in vacuum, Friedmann models in cosmology, Tolman-Bondi dust solutions and the Vaidya space-time in the case of non vacuum solutions.

In order to solve field equations basically one can use either g-method or T-method. In g-method metric functions $g_{a b}$ are assigned and then the energy momentum tensor $T_{a b}$ is obtained by mere differentiation via field equations. Thus in this regard every metric is an exact solution of the field equations. The problem with this method is that resulting $T_{a b}$ may and very likely do not describe a physically realistic distribution of matter. The Tmethod is most promising in the sense that one starts with a physically realistic situation by prescribing a suitable $T_{a b}$ and the field equations are then integrated to obtain the geometry of the spacetime via $g_{a b}$. However due to the high degree of non linearity of the differential equations involved, in very few cases exact solutions of the field equations has been obtained using this method. In this paper we wish to analyze the field equations from this perspective by prescribing stress energy tensor and use the T-method to obtain solutions of the field equations.

The vacuum Schwarzschild solution gives the gravitational field outside a spherically symmetric distribution of matter. However most of the stars radiate energy in the form of lightlike particles and thus the immediate space outside the star is filled with electromagnetic radiations (photon ). The Vaidya spacetime ${ }^{2}$ is used to describe the field of a radiating star. The stress energy tensor in Vaidya solution is of the form

$$
T^{a b}=\sigma k^{a} k^{b}
$$

which describes only the radially directed radiations. $\sigma$ is regarded as the energy flux density of the directed radiations in some frame depending on the normalization the vector $k^{a}$ which is tangent to null geodesics. In spherically symmetric spacetime it can be regarded as outgoing radiations with momentum only in radial direction and with $\sigma=p, p$ being the radial pressure. Vaidya spacetime had been quite extensively used not only to study stellar objects but also in connection with spherical collapse and formation of naked singularities ${ }^{3}$. However, in nature the space outside radiating stars is not filled only by the directed radiations but in fact there is a presence of isotropic radiations surrounding the immediate space out side the star. Such a gas of photons (isotropic radiations) may originate either from the star itself or from some outside sources. The energy density of such a photon gas (isotropic radiations) may be small or may even be decreasing much faster than the flux of directed radiations, which reach the distant observers.

The energy momentum tensor of the Vaidya spacetime is actually part of a larger class of energy momentum tensors which have a vanishing stress energy scalar namely $T=T_{a}^{a}=0$. In fact type I matter fields ${ }^{4}$, which account for all the known and observed physically realistic fields (except directed radiations which is type II), with a vanishing $T$ can be expressed as mixture of both isotropic and directed radiations as we point out in this paper. The aim 
of this paper is to analyze the field equations for spherically symmetric matter distributions with vanishing stress energy scalar and to find solutions in such cases where spacetime is filled with isotropic as well as directed radiations.

Thus the solutions obtained in this paper could be significant in the context of a gravitational collapse scenario. It is believed that in the late stages of collapse matter may disintegrate due to extreme temperature, densities and pressures into photons or lightlike particles in the form of a gas of lightlike particles. This may consist of both or either isotropic and anisotropic radiations. It could be argued that this photon gas may then disperse and thus no singularity may form as a result of collapse. We do, however, find in this paper that certain exact solutions describing a photon cloud as considered here may still develop a singularity in the spacetime. As expected all the static photon gas solutions with only isotropic radiations do not match with the Schwarzschild vacuum solution but interestingly some solutions representing a mixture of a isotropic photon gas and directed radiations as considered in this paper can be matched to the vacuum exterior only at the sphere of radius $14 M_{s} / 4$ where $M_{s}$ is the Schwarzschild Mass of the photon cloud.

The paper is organized as follows. In section II we consider the basic field equations for our model of spacetime describing the spherically symmetric matter distribution with vanishing $T$. A procedure for solving and obtaining solutions of the field equations with detailed discussion on type I fields is given in this section. In Section III we give a method for generating exact solutions of the field equations in this scenario. We also explore some explicit exact solutions describing various density distributions of the photon cloud in this section. Section IV is devoted to the matching conditions of solutions thus obtained to possible exterior metric out side the radiation zone. We discuss in appendix A the stress energy tensor with vanishing $T$ and point out that the same can be expressed as a mixture of isotropic radiations with diffusive terms as well as directed radiations. Appendix B very briefly gives the geometric quantities associated with the metric written in null coordinates.

\section{Field Equations and Radiation distributions}

Stress energy tensor of a spherically symmetric spacetime with a vanishing stress energy scalar $T=T_{a}^{a}=0$ (Ricci curvature scalar $R=0$ ) can always be expressed as (see appendix A)

$$
T^{a b}=T_{(\text {pht gas })}^{a b}+T_{(\text {dirct rad })}^{a b}+T_{(\text {diffusi })}^{a b}
$$

where

$$
\begin{gathered}
T_{(\text {pht gas })}^{a b}=(\rho+p) v^{a} v^{b}+p g_{a b}, \quad p=\frac{\rho}{3} \\
T_{(\text {dirct rad })}^{a b}=\sigma k^{a} k^{b} \\
T_{(\text {diffusi })}^{a b}=q^{a} v^{b}+q^{b} v^{a}
\end{gathered}
$$

Here $T_{\text {(pht gas) }}^{a b}$ represents the stress energy tensor of the isotropic radiations (photon gas) ${ }^{5}$, $\rho$ is the energy density of the isotropic radiations as measured in the rest frame of the timelike vector $v^{a}$ (i.e. $v^{a} v_{a}=-1$ ). $T_{\text {(dirct rad) }}^{a b}$ is the energy tensor of the directed radiations with vector $k^{a}$ tangent to null geodesics and $\sigma$ is the energy density of the directed radiations. $q^{a}$ is a spacelike vector normal to the timelike vector $v^{a}$ and is treated as the heat flux vector and $T_{(\text {diffusi) }}^{a b}$ represent contribution of diffusive terms in the stress energy tensor. 
Using the spherical symmetry and adopting the coordinates such that $k^{a} \propto \delta_{1}^{a}$ (i.e. null coordinates) the metric describing the space-time is then given by

$$
d s^{2}=-A d u^{2} \pm 2 e^{2 \beta} d u d r+r^{2}\left(d \theta^{2}+\sin ^{2} \theta d \phi^{2}\right), \quad u, r, \theta, \phi=0,1,2,3
$$

Vector $k_{a}=\delta_{a}^{0}$ is the propagation vector of the directed radial radiations, and $A=A(u, r)$ and $\beta=\beta(u, r)$ are functions of $u$ and $r, \pm$ relate to the use of either retarded of advanced time and is used to distinguish ingoing or outgoing radiations.

Stress energy tensor has only $T_{00}, T_{01}, T_{11}$ and $T_{33}=\sin ^{2} \theta T_{22}$ as non vanishing components. Since $T_{a}^{a}=0$ field equations for the space-time described by the metric (5) basically reduce to the following four equations

$$
R_{00}=k T_{00}, \quad R_{11}=k T_{11}, \quad R_{22}=k T_{22}, \quad R=0
$$

where $k$ is the gravitational constant and $R$ is the Ricci scalar curvature. Once the above equations are satisfied equation $R_{01}=k T_{01}$ is automatically satisfied. Using the metric (5), field equations (6) become (see appendix B for various geometric quantities and expressions for Ricci tensor)

$$
\begin{gathered}
k T_{11}=\frac{4 \beta^{\prime}}{r} \\
k T_{22}=1-e^{-2 \beta}\left(r A e^{-2 \beta}\right)^{\prime} \\
k T_{00}=\mp e^{2 \beta}\left(\frac{A e^{-4 \beta}}{r}\right) \pm A e^{-2 \beta} \dot{\beta}^{\prime}+\frac{A e^{-4 \beta}}{2}\left(A^{\prime \prime}+A^{\prime}\left(\frac{4}{r}-2 \beta^{\prime}\right)\right) \\
R=0 \Rightarrow r^{2} A^{\prime \prime}+2 r A^{\prime}\left(2-r \beta^{\prime}\right)+2 A\left(1-4 r \beta^{\prime}\right)=2 e^{4 \beta} \mp r^{2} e^{2 \beta} 4 \dot{\beta}^{\prime}
\end{gathered}
$$

where (') and (') represent partial derivatives with respect to $u$ and $r$ respectively. The procedure for solving the above field equations is straight forward as follows. Since there are only four equations to be satisfied and there are five unknowns namely $A, \beta, \rho, \sigma$ and $q^{2}=q^{a} q_{a}$ there is a degree of freedom in the choice of one function. From equation (1) and (7) follows that $\beta^{\prime}$ is related to the density of isotropic radiations $\rho$ and the $q$. Therefore in general one chooses $\beta=\beta(u, r)$ in a way desired for suitable density distribution of the photon gas or otherwise as the case may be. Equation (10) which is a second order ordinary differential for function $A$ in variable $r$ is then solved to find $A=A(u, r)$. The rest of the remaining three equations (7) to (9) then determine the three unknowns $T_{00}, T_{11}$ and $T_{22}$, which give the energy density of the isotropic radiations $\rho$, energy density of the directed radiations $\sigma$ and the heat flux $q$.

We wish to discuss mainly in this paper type I spherically symmetric matter fields ${ }^{4}$ in the context of exact solutions, since all observed fields are of this type except for type II which represent directed radiations only. Any type I matter field (i.e. $T^{a b}$ ) with vanishing scalar $T$ can be cast as (see equation (70) in appendix A)

$$
T^{a b}=\frac{4 \rho}{3} v^{a} v^{b}+\frac{\rho}{3} g^{a b}+\sigma k^{a} k^{b}
$$

except the special cases where the eignvalues corresponding to the eignvectors in the radial two space are equal in magnitude(see equations (71) and (72) in appendix A). We would 
mention here for the sake of completeness that only other physically reasonable matter fields are of type II and the stress energy tensor for this case is either Vaidya null dust given in equation (73) or photon gas with diffusion terms as given in equation (74). All other types of matter fields are not considered physically reasonable as they necessarily violate energy conditions.

We would focus our discussion on type I unless specified otherwise and we would use stress energy tensor given in (11) when giving explicit exact solutions. The stress energy tensor $T^{a b}$ in (11) represents type I matter fields and the weak energy conditions are satisfied provided

$$
\rho \geq 0, \quad \sigma \geq 0
$$

Let us now consider the field equation for a cloud of gas consisting of both isotropic and directed radiations as described by the stress energy tensor given in (11). For space-time given by $(5)$ timelike vector $v_{a}=\left(v_{0}, v_{1}, 0,0\right)\left(v_{1} \neq 0\right)$ satisfies

$$
A e^{-4 \beta}\left(v_{1}\right)^{2} \pm 2 e^{-2 \beta} v_{0} v_{1}=-1
$$

Note that for $T^{a b}$ as in (11) function $\beta^{\prime}$ is related to the density distribution of isotropic radiations in the space-time, for example if $\beta^{\prime}=0$ then from equation (7) and (11) it follows that $T_{11}=0 \rightarrow \rho=0$ and the cloud does not have any isotropic photon gas and is all directed radiations and the solution is given by Vaidya,s radiating star ${ }^{2}$. Hence one chooses $\beta^{\prime}$ as required for the suitable density distribution of the isotropic radiations and integrates equation (10) to find $A$. The energy density of the isotropic photon gas as measured in the rest frame of the timelike vector $v^{a}$ and of the directed radiations in the photon cloud are then given by equations (7) to (9) in terms of $\beta$ and $A$. We further have for $\rho, \sigma$, and $v_{1}$ using equations (5), (11), (13) and (7) to (10) in terms of $A$ and $\beta$

$$
\begin{gathered}
\frac{k \rho r^{2}}{3}=k T_{22}=1-e^{-2 \beta}\left(r A e^{-2 \beta}\right)^{\prime} \\
k \rho\left(v_{1}\right)^{2}=\frac{3}{4} k T_{11}=\frac{3 \beta^{\prime}}{r} \\
\sigma=T_{00}-\frac{\rho}{3}\left(4 v_{0}^{2}-A\right)= \pm e^{2 \beta} \frac{\left(r A \dot{e}^{-4 \beta}\right)}{k r^{2}}-\frac{\rho}{3 g^{11}\left(v_{1}\right)^{2}}\left(1-\left(g^{11}\left(v_{1}\right)^{2}\right)^{2}\right)
\end{gathered}
$$

\section{Exact Solutions}

As mentioned in the preceding section, one can solve the field equations by specifying the function $\beta(u, r)$ and integrating the ordinary second order linear differential equation ( $10)$ to get $A(u, r)$. Rest of the quantities are then calculated by differentiating $\beta$ and $A$ from equations (7) to (9) and (13).

The simple procedure to find an exact solution is as follows. Consider the homogeneous part of the differential equation (10) for $A=A(u, r)$ which is given by

$$
y^{\prime \prime}+\frac{y^{\prime}}{r}\left(4-2 r \beta^{\prime}\right)+\frac{2 y}{r^{2}}\left(1-4 r \beta^{\prime}\right)=0
$$

This is an ordinary second order linear differential equation and variable $u$ and functions of $u$ which appear in $\beta^{\prime}(u, r)$ are treated as constant in the differential equation. Let $y=y_{1}(r)$ be 
a solution of the differential equation (17). The general solution $A=A(u, r)$ of differential equation (10) is then given by

$$
\begin{gathered}
A(u, r)=M_{1}(u) y_{1}+M_{2}(u) y_{2}+A_{p} \\
y_{2}=y_{1} \int \frac{e^{2 \beta}}{r^{4} y_{1}^{2}} d r \\
A_{p}(u, r)=y_{1} \int\left(\int\left(e^{2 \beta} r^{2} y_{1} \mp r^{4} \dot{\beta}^{\prime} y_{1} d r\right) \frac{e^{2 \beta} d r}{\left(r^{2} y_{1}\right)^{2}}\right.
\end{gathered}
$$

Where $M_{1}(u)$ and $M_{2}(u)$ are arbitrary functions of $u, y_{2}$ is another solution of the homogeneous equation and $A_{p}$ represents the particular solution of equation (10). Thus $A(u, r)$ in above equation gives the general solution of the field equations for a given $\beta=\beta(u, r)$. In case $\beta^{\prime}=0$ the solution above reduce to charged null dust solution ${ }^{6}$ (i.e. charged Vaidya solution)

An interesting way to generate exact solutions for different density distributions is by selecting the function $y_{1}=y_{1}(u, r)$ and solving for $\beta$ in equation (17) that is

$$
\begin{gathered}
r \beta^{\prime}=\frac{y_{1}^{\prime \prime}+\frac{4 y_{1}^{\prime}}{r}+\frac{2 y_{1}}{r^{2}}}{\frac{2 y_{1}^{\prime}}{r}+\frac{8 y_{1}}{r^{2}}} \\
\Rightarrow e^{2 \beta}=\frac{r y^{\prime}+4 y}{r} \exp \left(\int \frac{3 d r}{r\left(r y^{\prime}+4\right)}\right)
\end{gathered}
$$

Thus for any given $y_{1}(u, r) \beta$ is known from the above equation (21) and then $A$ is given by the equation (18). Rest of the unknowns namely $T_{00}, T_{11}, T_{22}$ are immediate from equations (7) to (9) for the general $T^{a b}$ in (1), and in case of type I matter field $\rho(u, r), v_{1}$, and $\sigma(u, r)$ are given by (14) to (16).

We now consider few examples of exact solutions of physical interest for a photon cloud given by the energy tensor in (11) and to clarify the procedure.

1. $y_{1}=r^{n}, \quad n \neq-4$

Let us put $y_{1}=r^{n}$, we get $\beta$ from (22)

$$
e^{\beta(u, r)}=\left(\frac{r}{r_{o}}\right)^{m}, \quad m=\frac{(n+1)(n+2)}{2(n+4)}
$$

Equations (19) and (20) give

$$
\begin{gathered}
y_{2}=r^{l}, \quad l=-\frac{4 n+10}{n+4} \\
A_{p}=\frac{2 e^{4 \beta}}{(2 m+n+3)(4 m-n)} \mp \frac{e^{2 \beta}\left(n^{2}+8 n+10\right) \dot{n}}{2(n+4)^{2}}
\end{gathered}
$$

- The general solution for $A$ is then

$$
A(u, r)=M_{1}(u) y_{1}+M_{2} y_{2}+\frac{2 e^{4 \beta}}{a_{o}} \mp b_{o} e^{2 \beta}
$$




$$
a_{o}=(2 m+n+3)(4 m-n), \quad b_{o}=\frac{\left(n^{2}+8 n+10\right) \dot{n}}{2(n+4)^{2}}
$$

The energy density of the isotropic and directed radiations are

$$
\begin{gathered}
\frac{k \rho r^{2}}{3}=1-\left(M_{1}(u)(n-2 m+1) y_{1} e^{-4 \beta}+M_{2}(u)(l-2 m+1) y_{2} e^{-4 \beta}\right. \\
\left.+\frac{2(4 m+1)}{a_{o}} \mp e^{-2 \beta} b_{o}\right) \\
k \sigma= \pm \frac{e^{2 \beta}}{r}\left(y_{1}\left(M_{1}(\dot{u}) e^{-4 \beta}\right)+y_{2}\left(M_{2}(\dot{u}) e^{-4 \beta}\right)+\left(\frac{\dot{2}}{a_{o}}\right) \mp\left(b_{o} e^{-2 \beta}\right)\right) \\
-\frac{k^{2} \rho^{2}}{9 m g^{11}}\left(1-\left(\frac{3 m g^{11}}{k \rho r^{2}}\right)^{2}\right) \\
\left(v_{1}\right)^{2}=\frac{3 m}{k \rho r^{2}}
\end{gathered}
$$

Thus the solution involves four arbitrary functions of $u$ namely $M_{1}(u), M_{2}(u), n(u)$ and $r_{o}(u)$. We next consider few solutions for a given $\beta(u, r)$

2. $\beta=-\mu(u) / r^{n}$

In this section we mainly wish to obtain exact solutions which are either asymptotically flat in the sense that the metric becomes Minkowskian as $r \rightarrow \infty$ or reduce to Vaidya spacetime for $r>>0$. Furthermore these spacetime would reduce to Vaidya space-time in case $\mu=0$ as the isotropic energy density of photon gas would vanish. We give below some exact solutions for different configurations. As pointed out above, once a solution of the homogeneous equation (17) given as $y=y_{1}(r)$, the specification of all the unknowns namely $\rho, \sigma, A, v_{1}$ as pointed out earlier, becomes immediate from equations (13) to (16) and (18). Hence we give below few examples of exact solutions thus obtained.

$$
\begin{gathered}
\text { a) } \beta=-\frac{-\mu}{r}, \quad y_{1}=\frac{1}{r^{2}}\left(1+\frac{2 \mu}{r}+\frac{2 \mu^{2}}{3 r^{2}}\right) \\
\text { b) } \beta=-\frac{-\mu}{r^{2}}, \quad y_{1}=\frac{1}{r^{2}}\left(1+\frac{4 \mu}{r^{2}}\right) \\
\text { c) } \beta=-\frac{-\mu}{r^{3}}, \quad y_{1}=\frac{1}{r}\left(1+\frac{3 \mu}{r^{3}}\right)
\end{gathered}
$$

For a general form of $\beta$ which is vanishing as $r \rightarrow \infty$ one can find the power series solution of the homogenous equation (17) in the form of $y=y_{1}(r)$. The solution of the field equation is then immediate by the determination of $A$ from equations (18) which is given in terms of $\beta(u, r)$ and $y_{1}$.

\section{Static Photon Cloud}

For static solutions $\beta=\beta(r)$ and $A=A(r)$ hence in all previous solutions reduce to static solutions by putting function depending on $u$ as constants. However in static case 
energy densities of both the directed and isotropic radiations are related as we obtain from equation (16)

$$
k \sigma=\frac{m g^{11}}{r^{4}}-\frac{(k \rho)^{2}}{9 m g^{11}}
$$

In cases where the photon cloud does not contain any isotropic radiations and is static note that in such cases $\rho=0$ implies from (16) $\sigma=0$ and the spacetime does not have any radiations. We next consider exact solutions in cases where the radiation cloud does not consists of any directed radiations ${ }^{8}$ and is static i.e. $\dot{\beta}=\dot{A}=0$. Requiring additionally that energy density of the directed radiations $\sigma=0$ we get from equation the above equation for that

$$
\sigma=0=>1-g^{11}\left(v_{1}\right)^{2}=0
$$

Hence from equations (10) and (13) to (16) we get

$$
\begin{gathered}
e^{3 \beta}=Y^{\prime}, \quad A=\frac{Y e^{\beta}}{r} \\
Y^{\prime \prime} Y^{3}=c_{o} r^{4}\left(Y^{\prime}\right)^{\frac{4}{3}}
\end{gathered}
$$

where $c_{o}$ is a constant. Thus any solution of equation (35) gives a solution representing a cloud of static photon sphere (pure isotropic radiations). For one such solution note that $Y=$ const. $r^{\frac{7}{4}}$ satisfies equation (36) and hence we get the solution as

$$
\begin{aligned}
& e^{\beta}=\left(\frac{r}{r_{o}}\right)^{\frac{1}{4}} \\
& A=\frac{4 r}{7 r_{o}} \\
& \rho=\frac{3}{7 k r^{2}}, \quad \sigma=0, \quad v^{a}=\sqrt{\frac{7 r_{o}}{4 r}} \delta_{o}^{a}
\end{aligned}
$$

where $r_{o}$ is a constant.

\section{Boundary of the Photon Cloud}

The radiation zone described by the metric in (5) can be made continuous with either the Schwarzschild metric describing the vacuum space-time outside the radiation zone or with the Vaidya metric describing the zone of only directed radiations. In both cases we will express the exterior also in null coordinates

$$
d s^{2}=-\left(1-\frac{2 M(u)}{r}\right) d u^{2} \pm 2 d u d r+r^{2}\left(d \theta^{2}+\sin ^{2} \theta d \phi^{2}\right)
$$

For Schwarzschild space-time $M(u)=M_{s}$ where $M_{s}$ is a constant and denotes Schwarzschild mass. If $\Sigma$ is the three surface boundary separating the two regions of space-time than junction conditions have to be satisfied across the boundary. The jump conditions ${ }^{7}$ basically require that the metric $g_{a b}$ and the energy flux vector $T_{a b} n^{b}$ be continuous across the boundary $\Sigma$ to which $n^{a}$ is the normal vector. 
We would limit our consideration of jump conditions only across the three surfaces $u=$ constant $=T$ and $r=$ constant $=r_{c}$ keeping in mind that exterior metric is either Schwarzschild or Vaidya. Below we consider matching conditions across the boundary $r=$ const. which is spacelike and the null boundary at $u=$ const.

A. $\Sigma=r-r_{c}=0$

The junction conditions across the boundary $r=r_{c}=$ const., for the exterior metric tobe the either Vaidya metric consisting of only directed radiations or vacuum Schwarzschild imply that both the metric and the energy momentum flux be continuous across $r=r_{c}$. We have

$$
\Sigma=r-r_{c}=0 \Rightarrow n_{a}=\frac{1}{\sqrt{g^{11}}}(0,1,0,0)
$$

Continuity of metric for the form of metric in null coordinates given in (5) implies

$$
[A]_{r=r_{c}}=[C], \quad[\beta]_{r=r_{c}}=[C]
$$

where $[C]$ means continuity. The requirement that flux $T^{a b} n_{b}$ be continuous implies

$$
\frac{1}{\sqrt{g^{11}}} T_{0}^{1}=[C], \quad \frac{1}{\sqrt{g^{11}}} T_{1}^{1}=[C]
$$

We now consider the cases when exterior metric is described by either Vaidya or Schwarzschild solutions

\section{a. Exterior Vaidya metric}

The junction conditions across the boundary $r=r_{c}$ given ( 42) and (43) above in case of exterior metric being Vaidya space-time become

$$
\begin{gathered}
{[A]_{r=r_{c}}=1-\frac{2 M(u)}{r_{c}}, \quad[\beta]_{r=r_{c}}=0} \\
{\left[(r A)^{\prime}\right]_{r=r_{c}}=1, \quad\left[\left(A \dot{e}^{-4 \beta}\right)\right]_{r=r_{c}}=\left[\frac{-2 \frac{d M}{d v}}{r_{c}}\right]}
\end{gathered}
$$

Since these conditions require the continuity of the derivatives of functions with respect to $r$ and therefor it is not straight forward to obtain matching conditions in general, hence we take the exact solution where $\beta$ given by equations (23) and exact solution by equations (24) to (26) with $n=$ const., $r_{o}=$ const.. The junction condition imply

$$
\begin{gathered}
r_{c}=r_{o} \\
M(u)+E(u)+\frac{2}{a_{o}}=1-\frac{2 M(u)}{r_{c}} \\
n M(u)+l E(u)+\frac{8 m}{a_{o}}=\frac{2 M}{r_{c}}
\end{gathered}
$$

where we have put $M(u)=\left(r_{o}\right)^{n} M_{1}(u)$ and $E(u)=\left(r_{o}\right)^{l} M_{2}(u)$. The above equations relate the functions $M(u), E(u)$ to the Vaidya mass $M(u)$.

\section{b. Photon Gas Sphere in Vacuum}

We consider now the case of the static solution representing a photon cloud and see whether the junction condition across the boundary $r=$ const. $=r_{c}$ of the static photon 
cloud can satisfied for a possible exterior Schwarzschild metric given in (38) with $M(u)=$ const. $=M_{s}$. The O'Brine and Synge junction condition given by (42) and (43) become

$$
\begin{gathered}
{[A]_{r=r_{c}}=1-\frac{2 M_{s}}{r_{c}}, \quad[\beta]_{r=r_{c}}=0} \\
{\left[(r A)^{\prime}\right]_{r=r_{c}}=1}
\end{gathered}
$$

Thus static solutions can be matched if the above are satisfied. As an illustration let us consider a simple exact solution given in equations (23) to (26) with $n=M_{2}(u)=0$ and $r_{o}=$ const., $M_{1}(u)=$ const. $=M_{1}$ we have $A$ and $\beta$ for such a solution

$$
e^{2 \beta}=\left(\frac{r}{r_{o}}\right)^{\frac{1}{2}}, \quad A=M_{1}+\frac{4 r}{7 r_{o}}
$$

The junction conditions given above are satisfied

$$
r_{c}=r_{o}, \quad M_{1}=-\frac{1}{7}, \quad r_{o}=\frac{14 M_{s}}{4}
$$

Before closing the discussion on junction conditions we briefly mention that all static solutions with only isotropic photon gas as given by equations (36) to (38) do not satisfy the junction conditions above as expected. Thus although a pure isotropic photon gas sphere can not exist in a vacuum exterior, a mixture of directed radiation and isotropic photon gas can be matched to the Schwarschild vacuum solution at a sphere of radius $14 M_{s} / 4$. This means that a mixture of both isotropic and directed radiations need not necessarily diffuse through vacuum.

B. $\Sigma=u-T=0$, boundary of the radiation zone

Let us consider the boundary of the radiation zone at $u=T=$ constant and exterior metric tobe Schwarzschild. The junction conditions then require that at the boundary $\Sigma=$ $u-T=0$ with normal $n_{a}=(1,0,0,0)$ the metric be continuous which means

$$
[A]_{u=T}=\left(1-\frac{2 M_{s}}{r}\right), \quad[\beta]_{u=T}=0
$$

and the requirement that flux be continuous implies

$$
\left[T_{r r}\right]_{u=T}=0 \Rightarrow\left[\beta^{\prime}\right]_{u=T}=0, \quad\left[T_{u r}\right]_{u=T}=0 \Rightarrow\left[(r A)^{\prime}\right]_{u=T}=1
$$

Thus the junction conditions basically put restrictions on the choice of the function $\beta$. The metric in (5) is continuous with the exterior Schwarzschild space-time across the boundary $u=T$ provided $[\beta]_{u=T}=\left[\beta^{\prime}\right]_{u=T}=0$. This implies from equations (9) and (17) that at the boundary one of the solution of the homogeneous equation is $\left[y=y_{2}(u, r)\right]_{u=T}=\frac{1}{r}$ while the other $\left[y=y_{1}(u, r)\right]_{u=T}=\frac{1}{r^{2}}$ and thus (18) gives $A$ at the boundary

$$
[A]_{u=T}=1+\frac{M_{1}(T)}{r^{2}}+\frac{M_{2}(T)}{r}
$$

and therefore the boundary conditions imply

$$
M_{2}(T)=-2 M_{s}, \quad M_{1}(T)=0
$$


Thus all the solutions with function $\beta(u, r)$ such that $\beta(u=T, r)=\beta^{\prime}(u=T, r)=0$ the junction conditions are satisfied. For specific examples of exact solution given in equations (30) to (32) these imply that $\mu(T)=0$ and $M_{2}(T)=-2 M_{s}, \quad M_{1}(T)=0$

\section{APPENDIX A}

Because of the spherical symmetry one can decompose the spacetime metric as a combination of two 2-subspaces i.e. we can write

$$
\begin{gathered}
d s^{2}=g_{a b} d x^{a} d x^{b}=g_{i j} d x^{i} d x^{j}+R^{2}\left(d \theta^{2}+\sin ^{2} \theta d \phi^{2}\right) \\
x^{a}=\left(x^{i}, \theta, \phi\right), a=0,1,2,3 \quad i=0,1
\end{gathered}
$$

where $x^{i}, i=0,1$ are arbitrary coordinates which span the radial two space $(\theta, \phi)=$ const., $g_{i j}\left(x^{i}\right)$ is the metric of the radial two space and $R=R\left(x^{i}\right)$ describes the area of the two spheres $x^{i}=$ const..

Spherical symmetry imposes restrictions on the energy tensor $T^{a b}$. Thus there are two real spacelike eignvectors which lie in the two space for which $d x^{i}=0$ and the remaining in the two space for which $d \theta=d \phi=0$. In the former case the two eignvalues are equal. Thus the energy tensor $T^{a b}$ has only $T^{00}, T^{01}, T^{11}$, and $T_{\theta}^{\theta}=T_{\phi}^{\phi} \rightarrow \sin ^{2} \theta T^{\theta \theta}=T^{\phi \phi}$ as non vanishing components. Let $\left(p_{(\theta)}^{a}, p_{(\phi)}^{a}\right)$ be the two spacelike eignvectors of the stress energy tensor which lie in the two space $x^{i}=$ const. with eignvalues $\lambda_{\theta}$ we have

$$
p_{(\theta)}^{a}=\frac{1}{R} \delta_{\theta}^{a}, p_{(\phi)}^{a}=\frac{1}{R \sin \theta} \delta_{\phi}^{a}
$$

We further introduce a frame of two vectors which lie in the radial two space namely a timelike vector $v^{a}$ and a null vector $k^{a}$ such that

$$
v^{a} v_{a}=-1, \quad k^{a} k_{a}=k_{a} p_{(\theta)}^{a}=k_{a} p_{(\phi)}^{a}=v_{a} p_{(\theta)}^{a}=v_{a} p_{(\phi)}^{a}=0
$$

In general we can therefore express the stress energy tensor $T^{a b}$ as

$$
T^{a b}=A_{o} v^{a} v^{b}+B_{o} k^{a} k^{b}+C_{o}\left(k^{a} v^{b}+k^{b} v^{a}\right)+\lambda_{\theta} p_{(\theta)}^{a} p_{(\theta)}^{b}+\lambda_{\theta} p_{(\phi)}^{a} p_{(\phi)}^{b}
$$

where $\left(A_{o}, B_{o}, C_{o}\right)$ represent components of $T^{a b}$. Since the null vector lies in the two radial space we write $k^{a}=Q^{a}+v^{a}$ where $Q^{a}$ is a spacelike unit vector orthogonal to $v^{a}$. By writing

$$
k^{a} v^{b}+k^{b} v^{a}=2 v^{a} v^{b}+Q^{a} v^{b}+Q^{b} v^{a}
$$

and replacing $\left(k^{a} v^{b}+k^{b} v^{a}\right)$ in (60) we get $T^{a b}$ in the following form

$$
T^{a b}=A_{1} v^{a} v^{b}+B_{1} k^{a} k^{b}+C_{1}\left(Q^{a} v^{b}+Q^{b} v^{a}\right)+\lambda_{\theta} g^{a b}
$$

Requiring that $T_{a}^{a}=0$ we get

$$
A_{1}=4 \lambda_{\theta}
$$

and we can therefore express in general

$$
T^{a b}=\frac{4 \rho}{3} v^{a} v^{b}+\frac{\rho}{3} g^{a b}+\sigma k^{a} k^{b}+q^{a} v^{b}+q^{b} v^{a}
$$


where we have changed the notations as $\lambda_{\theta}=\frac{\rho}{3}, B=\sigma, q^{a}=C_{1} Q^{a}$ for the sake of physical reasons only.

The general matter fields described in (60) for spherically symmetric spacetime are physically realistic if they are either type I or II matter fields ${ }^{4}$. The type I fields are characterized by the existence of two distinct orthonormal real eignvectors in the radial two space while type II is characterized by the existence of one double null real eignvectors in the radial two space and can be written as

$$
\begin{gathered}
T^{a b}=\lambda_{0} E_{(0)}^{a} E_{(0)}^{b}+\lambda_{1} E_{(1)}^{a} E_{(1)}^{b}+\lambda_{\theta} p_{(\theta)}^{a} p_{(\theta)}^{b}+\lambda_{\theta} p_{(\phi)}^{a} p_{(\phi)}^{b} \\
T_{a b}=-2 \lambda_{I I} l_{(a} m_{b)}+m_{a} m_{b}+\lambda_{\theta} p_{(\theta)}^{a} p_{(\theta)}^{b}+\lambda_{\theta} p_{(\phi)}^{a} p_{(\phi)}^{b}
\end{gathered}
$$

where $E_{(0)}^{a}$ is the unit timelike eignvector othogonal to unit spacelike eignvector $E_{(1)}^{a}$ which lie in the radial two space with $\lambda_{o}$ and $\lambda_{1}$ as corresponding eignvalues in case of type I field in $(65)$ and $\left(l^{a}, m^{a}\right)$ are two real null vectors in the radial two space in case of type II fields in $(66)$.

All known observed physical fields are of type I except for the pure directed radiations which are the only known physically realistic fields belonging to type II fields. Therefore type I fields are of special interest. Let us consider a type I spherically symmetric matter fields given in (65). For matter cloud with a vanishing stress energy scalar $T=T_{a}^{a}=0$ imply

$$
\lambda_{1}-\lambda_{0}+2 \lambda_{\theta}=0 \Rightarrow \lambda_{1}=\lambda-\lambda_{\theta}, \quad \lambda_{o}=\lambda+\lambda_{\theta}
$$

In case the eignvalues in the radial two space are distinct that is $\lambda_{o} \neq \pm \lambda_{1}$ we could use a set of a null vector $k^{a}$ and a timelike vector $v^{a}$ in the radial two space such that

$$
\begin{gathered}
E_{(1)}^{a}=\sqrt{\frac{2 \lambda_{\theta}}{\lambda}} v^{a}+\frac{1}{2} \sqrt{\frac{2 \lambda_{\theta}}{\lambda}}\left(1+\frac{2 \lambda_{\theta}}{\lambda}\right) \frac{k^{a}}{k^{a} v_{a}} \\
E_{(o)}^{a}=-\sqrt{\frac{2 \lambda_{\theta}}{\lambda}} v^{a}+\frac{1}{2} \sqrt{\frac{2 \lambda_{\theta}}{\lambda}}\left(1-\frac{2 \lambda_{\theta}}{\lambda}\right) \frac{k^{a}}{k^{a} v_{a}}
\end{gathered}
$$

The stress energy tensor for type I fields then can be expressed as

$$
T^{a b}=\frac{4 \rho}{3} v^{a} v^{b}+\frac{\rho}{3} g^{a b}+\sigma k^{a} k^{b}
$$

where we changed the notation $\lambda_{\theta}=\frac{\rho}{3}$ for the sake of for physical reasons. In special cases where $\lambda_{0}= \pm \lambda_{1}$ we can cast $T^{a b}$ in (65) as

$$
\begin{gathered}
T^{a b}=\sigma\left(k^{a} k^{b}+q^{a} u^{b}+q^{b} u^{a}\right), \quad \text { for } \lambda_{0}=\lambda_{1} \\
T^{a b}=\frac{\rho}{3}\left(4 v^{a} v^{b}+g^{a b}+k^{a} k^{b}+q^{a} u^{b}+q^{b} u^{a}\right), \quad \text { for } \lambda_{0}=-\lambda_{1}
\end{gathered}
$$

where $k^{a}=q^{a}-v^{a}$ is a null vector, $v^{a}$ is a unit timelike vector which is orthogonal to spacelike unit vector $q^{a}$.

Though in this paper we would only deal with type I matter fields, however, for the sake of completeness of discussion we wish to mention that type II fields characterized by equation (66) can be expressed in case $\lambda_{I I}=0$ as

$$
T^{a b}=\sigma k^{a} k^{b}+\lambda_{\theta} p_{(\theta)}^{a} p_{(\theta)}^{b}+\lambda_{\theta} p_{(\phi)}^{a} p_{(\phi)}^{b}
$$


or in case $\lambda_{I I} \neq 0$

\section{Appendix B.}

$$
T^{a b}=\frac{4 \rho}{3} v^{a} v^{b}+\frac{\rho}{3} g^{a b}+\left(q^{a} v^{b}+q^{b} v^{a}\right)
$$

Here we give the necessary geometric quantities associated with the metric

$$
d s^{2}=-A d u^{2} \pm 2 e^{2 \beta} d u d r+r^{2}\left(d \theta^{2}+\sin ^{2} \theta d \phi^{2}\right), \quad u, r, \theta, \phi=0,1,2,3
$$

The non vanishing metric components and Christoffel symbols associated with the metric are

$$
\begin{gathered}
g^{11}=A e^{-4 \beta}, \quad g^{01}= \pm e^{-2 \beta}, \quad g^{22}=r^{2} \quad g^{33}=r^{2} \sin ^{2} \theta \\
\Gamma_{12}^{2}=\Gamma_{13}^{3}=\frac{1}{r}, \quad, \Gamma_{32}^{2}=\cot \theta, \Gamma_{33}^{2}=-\sin \theta \cos \theta \\
\Gamma_{22}^{1}=\sin ^{-2} \theta \Gamma_{33}^{1}=-r g^{11}, \quad \Gamma_{22}^{0}=\sin ^{-2} \theta \Gamma_{33}^{0}=-r g^{01} \\
\Gamma_{00}^{0}=2 \dot{\beta} \pm \frac{A^{\prime}}{2} e^{-2 \beta}, \Gamma_{11}^{1}=2 \beta^{\prime}, \quad \Gamma_{01}^{1}=\mp \frac{A^{\prime} e^{-2 \beta}}{2} \\
\Gamma_{00}^{1}= \pm\left(2 \dot{\beta} A-\frac{\dot{A}}{2}\right) e^{-2 \beta}+\frac{A^{\prime}}{2} A e^{-4 \beta}
\end{gathered}
$$

The non vanishing components of the Ricci Tensor are

$$
\begin{gathered}
R_{11}=\frac{4 \beta^{\prime}}{r} \\
R_{22}=1-e^{-2 \beta}\left(r A e^{-2 \beta}\right)^{\prime} \\
R_{00}= \pm e^{2 \beta}\left(\frac{A e^{-4 \beta}}{r}\right) \pm A e^{-2 \beta} \dot{\beta}^{\prime}+\frac{A e^{-2 \beta}}{2}\left(A^{\prime \prime} e^{-2 \beta}+2 A^{\prime} e^{-2 \beta}\left(\frac{2}{r}-2 \beta^{\prime}\right)\right) \\
R_{01}=-\dot{\beta}^{\prime} \mp \frac{e^{-2 \beta}}{2}\left(A^{\prime \prime}+2 A^{\prime}\left(\frac{2}{r}-2 \beta^{\prime}\right)\right) \\
R=\left(A^{\prime \prime}+\frac{2 A^{\prime}}{r}\left(2-r \beta^{\prime}\right)+\frac{2 A}{r^{2}}\left(1-4 r \beta^{\prime}\right)\right) e^{-4 \beta}-\frac{2}{r^{2}} \pm 4 \dot{\beta}^{\prime}
\end{gathered}
$$

\section{References}

1. D. Kramer, H. Stephani, E. Herlt and M. MacCallum, 1980, Exact Solutions of Einstein's Field Equations, Cambridge University Press.

2. P. C. Vaidya Nature 171, 260, (1953).

3. Dwivedi I H and Joshi P S 1989 Class Quantunm Grav 6 1599.;K. Lake Phys. Rev. D 43 1416(1991)

4. Hawking S W and Ellis G F R 1973 The Large Scale Structure of Space-Time Cambridge University Press. p. 88.

5. J. L. Synge, 1957 The Relativistic Gas (Amesterdam North Holland) p. 37; Thorne K. S. Mon. Not. R. astr. Soc 194, 439 (1981); Weinberg, S. Astrophys. J. 168, 175, (1972)

6. W. B. Bonor and P. C. Vaidya, Gen Relativ. Gravit. 1, 127, (1970); B T Sullivan and W Israel Phys. Lett. 79A, 371, (1980).

7. Synge J. L. 1961 Relativity: The General Theory (Amesterdam: North Holland) P.39

8. Perfect fluid solutions with $p=\rho / 3$ has been discussed in cosmological context such as Friedmann models. See also Klein. O. (1947) Ark. Mat. Astr. Pys. A 34 1. See 14.1. 